\title{
Escherichia coli and its lipopolysaccharide modulate in vitro Candida biofilm formation
}

Correspondence

L. P. Samaranayake

lakshman@hkucc.hku.hk

Received 21 May 2009

Accepted 5 August 2009

\author{
H. M. H. N. Bandara, J. Y. Y. Yau, R. M. Watt, L. J. Jin \\ and L. P. Samaranayake
}

Faculty of Dentistry, University of Hong Kong, Oral Biosciences, 5/F Prince Phillip Dental Hospital, 34 Hospital Road, Sai Ying Pun, Hong Kong SAR

\begin{abstract}
Demystification of microbial behaviour in mixed biofilms could have a major impact on our understanding of infectious diseases. The objectives of this study were to evaluate in vitro the interactions of six different Candida species and a Gram-negative coliform, Escherichia coli, in dual-species biofilms, and to assess the effect of E. coli LPS on Candida biofilm formation. A single isolate of E. coli ATCC 25922 and six different species of Candida, Candida albicans ATCC 90028, Candida glabrata ATCC 90030, Candida krusei ATCC 6258, Candida tropicalis ATCC 13803, Candida parapsilosis ATCC 22019 and Candida dubliniensis MYA-646, were studied using a standard biofilm assay. Each Candida species was co-cultured with E. coli on a polystyrene surface and biofilm formation was quantified by a c.f.u. assay. The biofilm was then analysed by Live/Dead staining and fluorescence microscopy (confocal laser-scanning microscopy, CLSM), whilst scanning electron microscopy (SEM) was employed to visualize the biofilm architecture. The effect of E. coli LPS on Candida biofilm cell activity at defined time intervals was assessed with an XTT reduction assay. A significant quantitative reduction in c.f.u. counts of C. tropicalis (after $90 \mathrm{~min}$ ), C. parapsilosis (after $90 \mathrm{~min}$ and $24 \mathrm{~h}$ ), C. krusei (after $24 \mathrm{~h}$ ) and $C$. dubliniensis (after 24 and $48 \mathrm{~h}$ ) was noted on incubation with $E$. coli in comparison with their monospecies biofilm counterparts $(P<0.05)$. On the other hand, a simultaneous and significant reduction in E. coli cell numbers occurred on co-culture with C. albicans (after $90 \mathrm{~min}$ ), and an elevation of $E$. coli cell numbers followed co-culture with $C$. tropicalis (after $24 \mathrm{~h}$ ) and $C$. dubliniensis (after $24 \mathrm{~h}$ and $48 \mathrm{~h})(P<0.05)$. All quantitative findings were confirmed by SEM and CLSM analyses. By SEM observation, dual-species biofilms demonstrated scanty architecture with reduced visible cell counts at all stages of biofilm development, despite profuse growth and dense colonization in their single-species counterparts. Significantly elevated metabolic activity, as assessed by XTT readings, was observed in E. coli LPS-treated C. tropicalis and C. parapsilosis biofilms (after $48 \mathrm{~h}$ ), whilst this had the opposite effect for C. dubliniensis (after $24 \mathrm{~h})(P<0.05)$. These data indicate that $E$. coli and Candida species in a mixed-species environment mutually modulate biofilm development, both quantitatively and qualitatively, and that E. coli LPS appears to be a key component in mediating these outcomes.
\end{abstract}

\section{INTRODUCTION}

In nature, most micro-organisms do not exist as pure species and prefer a lifestyle of community growth with one or more other species in a complex community organization termed a biofilm. By definition, a biofilm is a complex functional community of one or more species of microbes encased in an extracellular polymeric network and attached to one another or to a solid surface (Samaranayake, 2006).

Abbreviations: CLSM, confocal laser-scanning microscopy; SEM, scanning electron microscopy.

A table of c.f.u. counts of Candida species and E. coli from biofilms at different time intervals is available as supplementary data with the online version of this paper.
According to the US National Health Institute and the Centers for Disease Control and Prevention, it has been estimated that more than $60 \%$ of all microbial infections are associated with biofilms (Lewis, 2001; Potera, 1999). Some examples of biofilm-related infections include cystic fibrosis-related infections, catheter-related infections, periodontal diseases and middle ear infections (Douglas, 2003).

Candida, a common commensal fungus of humans, may convert to an opportunistic pathogen causing superficial or deep infections in susceptible hosts including neonates, patients undergoing immunosuppressive or broad-spectrum antibiotic therapy, or those with indwelling catheters, human immunodeficiency virus infection and diabetes (Odds, 1987). 
Indeed, Candida is considered the fourth most commonly isolated pathogen from bloodstream infections in US institutions (Banerjee et al., 1991; Jones et al., 1997; Pfaller et al., 1998) and these infections appear to have, overall, the highest crude mortality (Crump \& Collignon, 2000).

Constitutionally, a biofilm comprises either a single species (monospecies) or, more usually, multiple species of either bacteria alone or bacteria together with fungi such as Candida (multispecies) (Nobile \& Mitchell, 2007). The architecture and functionality of such multispecies biofilms are complex and the biofilms exhibit interspecies relationships that have yet to be unravelled, as the organisms coexist within a community with multifarious demands (Carlsson, 1997). Most of the work in the literature investigating such multispecies biofilms is based on multibacterial biofilms and there are few data on the interactions within fungal-bacterial biofilms. In one of the earliest studies, Nair \& Samaranayake (1996a) demonstrated that adhesion of Candida albicans to denture acrylic surfaces was promoted by Escherichia coli, a Gram-negative bacterium. In subsequent studies, they reported that this coliform significantly suppressed the adhesion of C. albicans and Candida krusei to human buccal epithelial cells (Nair \& Samaranayake, 1996b). More recently, the same group (Thein et al., 2006) confirmed that coliforms such as E. coli and Pseudomonas aeruginosa can significantly compromise C. albicans biofilm formation in a dose-dependent manner.

The endotoxin LPS, a cell-wall constituent of all Gramnegative bacteria including E. coli, possesses immunomodulatory and pro-inflammatory properties (Morrison \& Ryan, 1987; Rietschel et al., 1982). The effect of LPS on Candida was examined by Akagawa et al. (1995) and HenryStanley et al. (2003), who noted that Candida-associated mortality of experimental animals was augmented by E. coli and its LPS. It has been stated that LPS itself has no direct effect on candidal growth (Palma et al., 1992), but the immunomodulatory effects may be due to a secondary effect on polymorphonuclear leukocytes and monocytes (Cohn, 1978; Dahinden et al., 1983; Henricks et al., 1983). To date, the role of E. coli LPS on Candida-E. coli mixed biofilms has not been evaluated. Furthermore, most of the previous studies on interactions between Candida and bacteria in mixed biofilms focused only on C. albicans, and there are no studies to our knowledge relating to non-albicans Candida species biofilms in a mixed-species environment.

Hence, the aims of this study were to evaluate the interactions of E. coli and six different Candida species, C. albicans, Candida glabrata, Candida tropicalis, Candida parapsilosis, Candida dubliniensis and C. krusei, in dualspecies biofilms and to assess the effect of E. coli LPS on the biofilm development of these Candida species.

\section{METHODS}

Experimental design. The study comprised two components. First, a series of experiments was conducted to evaluate the combined effect of each of six different species of Candida and one isolate of E. coli on biofilm formation at three different time intervals. Secondly, a series of experiments was conducted to assess the effect of E. coli LPS on the biofilm development of the selected Candida species.

Micro-organisms. The following reference laboratory strains of Candida and E. coli were used: C. albicans ATCC 90028, C. glabrata ATCC 90030, C. tropicalis ATCC 13803, C. parapsilosis ATCC 22019, C. krusei ATCC 6258, C. dubliniensis MYA-646 and E. coli ATCC 25922. The identity of each organism was confirmed with the commercially available API 32C (for Candida strains) and API $20 \mathrm{E}$ (for E. coli) identification systems (bioMérieux). All isolates were stored in multiple aliquots at $-20{ }^{\circ} \mathrm{C}$, after confirming their purity.

LPS. LPS purified from E. coli O111: B4 (Ready Made solution, $1 \mathrm{mg}$ $\mathrm{ml}^{-1}, 0.2 \mu \mathrm{m}$ filtered) was purchased from Sigma Aldrich and stored at $2-8{ }^{\circ} \mathrm{C}$ until use.

Growth media. Sabouraud dextrose agar (SDA) and yeast nitrogen base (YNB) solution supplemented with $100 \mathrm{mM}$ glucose were used for culturing Candida species, and blood agar, MacConkey agar and tryptic soy broth (TSB) were utilized for E. coli culture.

Microbial inocula. Prior to each experiment, Candida species and $E$. coli were subcultured on SDA and blood agar, respectively, for $18 \mathrm{~h}$ at $37^{\circ} \mathrm{C}$. A loopful of this overnight growth was inoculated into YNB (Candida) or TSB (E. coli) medium and incubated for $18 \mathrm{~h}$ in an orbital shaker (75 r.p.m.) at $37{ }^{\circ} \mathrm{C}$. The resultant growth was harvested, washed twice in PBS and resuspended in PBS. PBS without calcium and magnesium ions was used to wash and rinse the suspended cells, as these ions facilitate cell binding and clumping, which would mask the mutual effects of Candida and E. coli during biofilm development. Concentrations of Candida species and E. coli were adjusted to $1 \times 10^{7}$ cells $\mathrm{ml}^{-1}$ by spectrophotometry and were confirmed by haemocytometric counting.

\begin{abstract}
Biofilm formation. Candida biofilms were developed as described by Jin et al. (2004) with some modifications. Commercially available presterilized, polystyrene, flat-bottomed, 96-well microtitre plates (Iwaki) were used. A standard cell suspension $(100 \mu \mathrm{l})$ of Candida species and E. coli $\left(10^{7}\right.$ cells $\mathrm{ml}^{-1}, 1: 1$ ratio) was prepared and transferred into selected wells of a microtitre plate. The plate was incubated for $1.5 \mathrm{~h}$ at $37{ }^{\circ} \mathrm{C}$ in an orbital shaker at 75 r.p.m. to promote microbial adherence to the surface of the wells. A volume of $100 \mu \mathrm{l}$ of monospecies controls of Candida species and E. coli was inoculated in an identical fashion. After the initial adhesion phase, the cell suspensions were aspirated and each well was washed twice with PBS to remove loosely adherent cells. A volume of $200 \mu$ TSB was added to each well and the plate was reincubated for 24 or $48 \mathrm{~h}$. The wells were then washed two or three times at respective time intervals with PBS to eliminate traces of TSB. The bacterial-fungal interactions were studied at $90 \mathrm{~min}$ (adhesion phase), $24 \mathrm{~h}$ (colonization phase) and $48 \mathrm{~h}$ (maturation phase) time intervals.
\end{abstract}

\section{Quantitative analyses}

Spiral plating and c.f.u. assay. At the end of the adhesion (90 min), colonization $(24 \mathrm{~h})$ and maturation $(48 \mathrm{~h})$ phases, after washing as described above, $100 \mu \mathrm{l}$ PBS was added to each well and the biofilm mass was meticulously scraped off the well wall using a sterile scalpel (Jin et al., 2004). The resultant suspension containing the detached biofilm cells was gently vortexed for $1 \mathrm{~min}$ to disrupt the aggregates, serially diluted and inoculated using a spiral plater onto SDA for Candida species and MacConkey agar for E. coli. The resultant c.f.u. $\mathrm{ml}^{-1}$ of yeast and bacteria were quantified after $48 \mathrm{~h}$ incubation at $37^{\circ} \mathrm{C}$. Each assay was carried out in triplicate on three different occasions. 
Evaluation of the effect of E. coli LPS using an XTT reduction assay. In the second part of the study, the effect of E. coli LPS on Candida species was evaluated. Freshly prepared LPS $\left(1 \mathrm{mg} \mathrm{ml}^{-1}\right.$, Ready Made solution; Sigma Aldrich) and YNB supplemented with $100 \mathrm{mM}$ glucose were mixed to yield a final concentration of $100 \mu \mathrm{g} \mathrm{LPS} \mathrm{ml}{ }^{-1}$ in the medium of the test sample. LPS was replaced with sterile PBS in the control. Candida biofilms were formed in three different 96-well plates to evaluate the effect of E. coli LPS at $90 \mathrm{~min}, 24 \mathrm{~h}$ and $48 \mathrm{~h}$ as described below.

First, Candida suspension medium $(100 \mu \mathrm{l}$ each) was decanted after centrifugation, resuspended in test and control medium (see above), dispersed into selected wells of a 96-well plate at time 0 and incubated in an orbital shaker ( 75 r.p.m.) at $37{ }^{\circ} \mathrm{C}$ for $90 \mathrm{~min}$ for the adhesion assay. In the second assay, $200 \mu \mathrm{l}$ test and control medium was added at $90 \mathrm{~min}$ to a different 96-well plate with pre-adherent Candida and incubated for $24 \mathrm{~h}$ for the initial colonization study. Finally, in the third assay, the latter procedure was repeated at $24 \mathrm{~h}$ for initially colonized Candida in a different 96-well plate and incubated for a further $24 \mathrm{~h}$ for the maturation phase study. At the end of the incubation period, the supernatant was removed and the wells were washed twice with sterile PBS to remove loosely adherent cells. A standard XTT reduction assay was performed, as described by Jin et al. (2004), to measure the metabolic activity of biofilms. In brief, commercially available XTT powder (Sigma) was dissolved in PBS to a final concentration of $1 \mathrm{mg} \mathrm{ml}^{-1}$. The solution was filter-sterilized $(0.22 \mu \mathrm{m}$ pore-size filter $)$ and stored at $-70{ }^{\circ} \mathrm{C}$. Freshly prepared $0.4 \mathrm{mM}$ menadione solution was used for the XTT reduction assay. XTT solution was thawed and mixed with menadione solution at a ratio of $20: 1(\mathrm{v} / \mathrm{v})$ immediately before the assay. Thereafter, $158 \mu \mathrm{l}$ PBS, $40 \mu \mathrm{l}$ XTT and $2 \mu \mathrm{l}$ menadione solution were added to each well containing pre-washed biofilms and incubated in the dark for $3 \mathrm{~h}$ at $37^{\circ} \mathrm{C}$. Colour changes were measured with a microtitre plate reader (SpectraMax 340 tunable microplate reader; Molecular Devices) at $492 \mathrm{~nm}$. All assays were carried out in triplicate on three different occasions.

Qualitative analyses Confocal laser-scanning microscopy (CLSM) (Jin et al., 2005) and scanning electron microscopy (SEM) were used to observe the ultrastructure of the Candida and E. coli biofilms.

CLSM. Commercially available pre-sterilized flat-bottomed six-well plates (Iwaki) and pre-sterilized plastic coverslips (Thermanox; Nulge Nunc International) (Ramage et al., 2001) were used to prepare biofilms as described above. Pre-sterilized coverslips were placed in the wells of a six-well plate, and suspensions of the monospecies or dual species were added. The plate was incubated for $90 \mathrm{~min}$ (adhesion phase) in an orbital shaker ( 75 r.p.m.) at $37{ }^{\circ} \mathrm{C}$. Thereafter, the supernatant was removed, the wells were washed twice with PBS, fresh TSB was added and the plate was incubated for $24 \mathrm{~h}$ (initial colonization) or $48 \mathrm{~h}$ (maturation phase) under the same environmental conditions. At the end of each time interval, the pre-washed coverslips were stained with Live/Dead stain (Live/Dead BacLight Bacterial Viability kit; Invitrogen) (Jin et al., 2005). The biofilm was then analysed by fluorescence microscopy (by CLSM).

SEM. For SEM, we grew single-species biofilms (Candida or E. coli alone), as well as Candida and E. coli mixed biofilms, on custommade, tissue culture-treated, polystyrene coverslips as described above. At $90 \mathrm{~min}, 24 \mathrm{~h}$ and $48 \mathrm{~h}$, selected coverslips were removed from the wells, washed twice with PBS and placed in $1 \%$ osmium tetroxide for $1 \mathrm{~h}$. Samples were subsequently washed in distilled water, dehydrated in a series of ethanol washes $(70 \%$ for $10 \mathrm{~min}$, $95 \%$ for $10 \mathrm{~min}$ and $100 \%$ for $20 \mathrm{~min}$ ) and air dried in a desiccator prior to sputter coating with gold. Specimens were mounted on aluminium stubs with copper tape and coated with gold under low pressure with an ion sputter coater (JFC1 100; JEOL). The surface topographies of the biofilm were visualized by SEM (Philips XL30CP) in high-vacuum mode at $10 \mathrm{kV}$ and the images were processed.
Statistical analysis Statistical analysis was performed using SPSS software (version 16.0). A Mann-Whitney $U$-test was performed to compare the significant differences between the control and each test sample of the bacterial-candidal biofilm. Data from all Candida species and E. coli analyses at different time points were pooled and evaluated using a Wilcoxon matched-pairs test. The percentage modulation of candidal biofilm growth in the presence of $E$. coli and E. coli LPS in pooled data was compared using a $\chi^{2}$ test. A value of $P$ $<0.05$ was considered statistically significant.

\section{RESULTS}

\section{Candida and $E$. coli dual-species biofilm growth (supplementary Table S1 in JMM Online)}

After $90 \mathrm{~min}$ of biofilm development with E. coli, a significant reduction in Candida cell counts was noted with C. tropicalis $(18 \%, P=0.010)$ and C. parapsilosis $(74 \%$, $P=0.030)$ compared with the controls, but this effect was not seen for C. albicans, C. glabrata, C. dubliniensis or C. krusei (Fig. 1a). Conversely, after $90 \mathrm{~min}$, a significant reduction in the number of $E$. colic.f.u. $(44 \%, P=0.047$ ) was observed in the presence of $C$. albicans but not with the other five Candida species (Fig. 1d).

After prolonged incubation for $24 \mathrm{~h}$, a significant reduction in the counts of C. krusei $(76 \%, P=0.050), C$. parapsilosis $(78 \%, P=0.011)$ and C. dubliniensis $(69 \%, P$ $<0.001)$ was noted in dual-species biofilms with $E$. coli (Fig. 1b). However, C. albicans, C. tropicalis and C. glabrata counts were unaffected. On the other hand, the mean number of $E$. coli c.f.u. increased significantly in the presence of $C$. tropicalis $(110 \%, P=0.019)$ and $C$. dubliniensis $(11 \%, P=0.017)$ after $24 \mathrm{~h}$, whilst the other four Candida species had no significant effect on E. coli numbers at this time point (Fig. 1e).

On further incubation for $48 \mathrm{~h}, C$. dubliniensis growth was highly suppressed in dual-species biofilms with E. coli (85\%, $P<0.001$ ), whilst the remaining Candida species were unaffected (Fig. 1c). Simultaneously, the mean c.f.u. of $E$. coli increased dramatically in co-cultures with $C$. tropicalis ( $170 \%, P=0.003)$ and moderately so with C. glabrata (50\%, $P=0.042$ ) (Fig. 1f). This effect on E. coli was not seen at this time point with the four remaining Candida species.

Despite these variable results at different time intervals, when data from all Candida species were pooled and analysed, a highly significant inhibition of Candida biofilm formation by E. coli was noted $(P<0.005)$. Furthermore, there appeared to be a simultaneous significant enhancement of $E$. coli biofilm development at 24 and $48 \mathrm{~h}$ time intervals triggered as a result of cohabitation with Candida $(P<0.005)$, particularly for $C$. tropicalis, C. dubliniensis and C. glabrata.

\section{Effect of E. coli LPS on Candida biofilms (XTT reduction assay)}

In the early biofilm development phase, after 90 min of incubation, none of the LPS-treated Candida species 
(a)

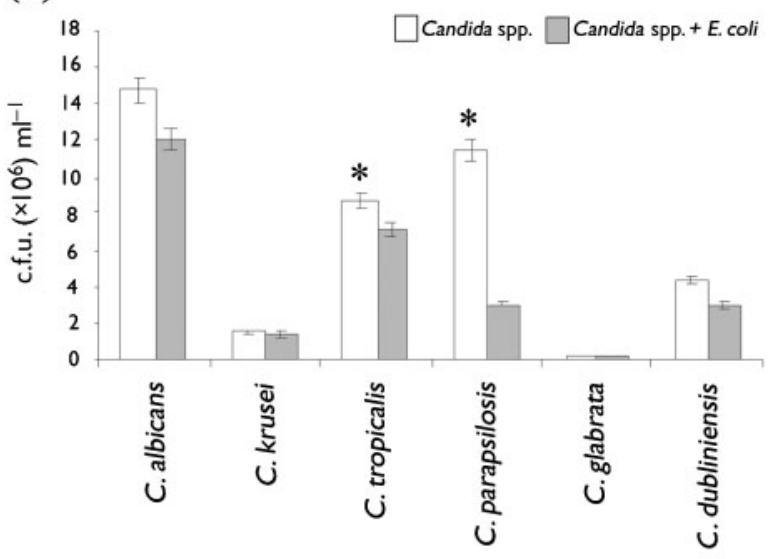

(b)

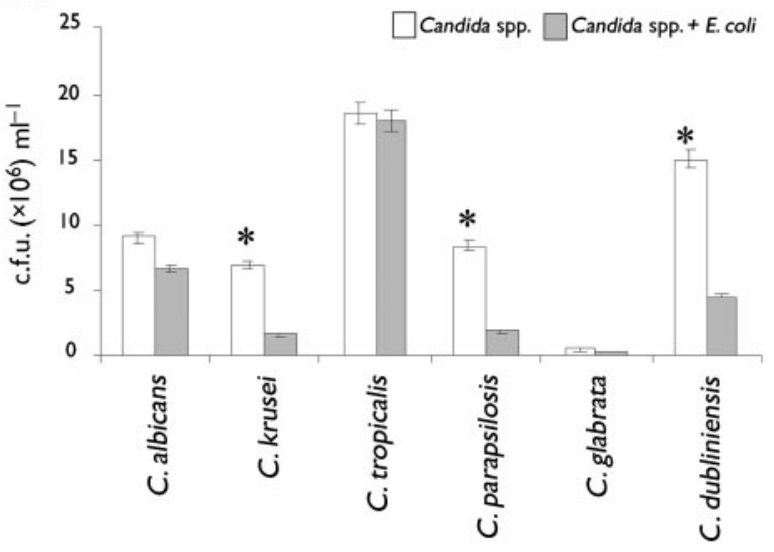

(c)

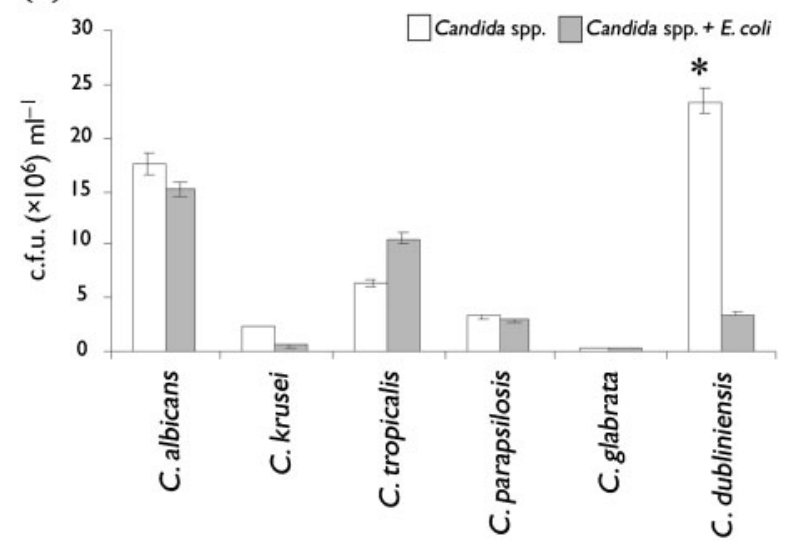

(d)

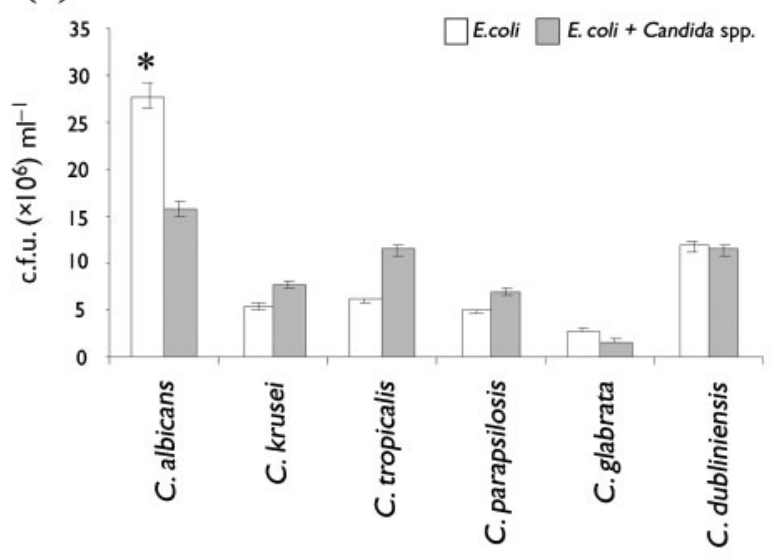

(e)

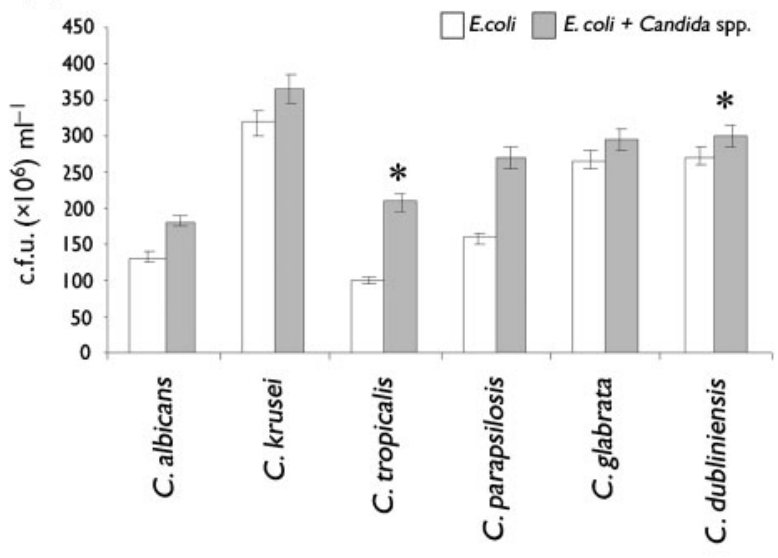

(f)

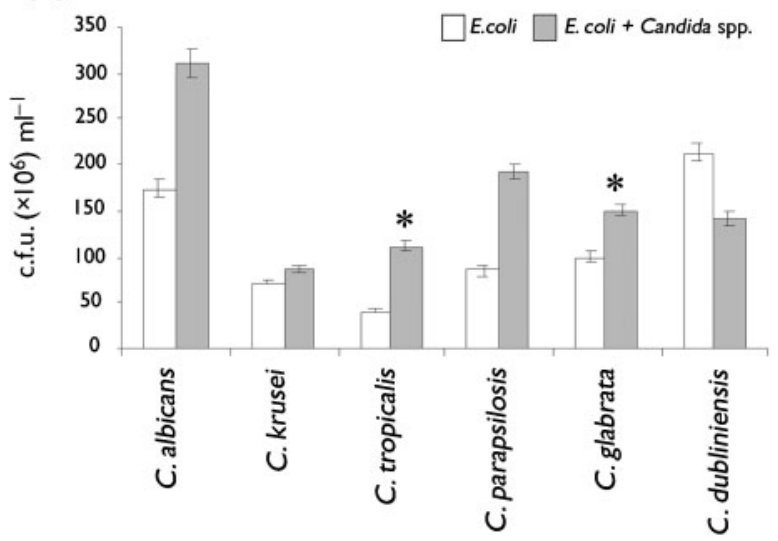

Fig. 1. Mean ( $\pm 5 \%$ ) c.f.u. counts of monospecies (Candida species or E. coli) and dual species (Candida species and E. coli) at different stages of biofilm formation. (a-c) Effect of $E$. coli on the biofilm formation of each Candida species at $90 \mathrm{~min}, 24 \mathrm{~h}$ and $48 \mathrm{~h}$, respectively. Asterisks indicate a significant $(P<0.05)$ reduction in mean c.f.u. counts of $C$. tropicalis $(P=0.010)$ and $C$. parapsilosis $(P=0.030)$ at $90 \mathrm{~min}(\mathrm{a})$, C. krusei $(P=0.050)$, C. parapsilosis $(P=0.011)$ and C. dubliniensis $(P<0.001)$ at $24 \mathrm{~h}(\mathrm{~b})$ and C. dubliniensis $(P<0.001)$ at $48 \mathrm{~h}(\mathrm{c})$ in the presence of $E$. coli. $(\mathrm{d}-\mathrm{f})$ Effect of each Candida species on the biofilm formation of $E$. coli at $90 \mathrm{~min}, 24 \mathrm{~h}$ and $48 \mathrm{~h}$, respectively. A significant reduction $(P<0.05)$ in mean c.f.u. counts of $E$. coli was noted in the presence of $C$. albicans $(P=0.047)$ at $90 \mathrm{~min}(\mathrm{~d})$ and a significant enhancement $(P<0.05)$ of mean c.f.u. counts of $E$. coli with $C$. tropicalis $(P=0.019)$ and $C$. dubliniensis $(P=0.017)$ at $24 \mathrm{~h}(\mathrm{e})$ and $C$. tropicalis $(P=0.003)$ and C. glabrata $(P=0.042)$ at $48 \mathrm{~h}$ (f). *, Significant difference between monospecies and dual-species biofilms at a given time point $(P<0.05)$. 
demonstrated significant changes in metabolic activity compared with the controls, indicating that LPS has minimal or no effect on the biofilm metabolism at this stage. However, after $24 \mathrm{~h}$, the metabolic activity of LPStreated C. tropicalis biofilms increased significantly (19\%, $P=0.008)$ in contrast to the biofilm activity of $C$. dubliniensis, which was significantly suppressed (4\%, $P=0.004$ ) (Table 1). LPS appeared to have no significant effect on the other four Candida species at this stage. On further incubation for $48 \mathrm{~h}$, only LPS-treated C. parapsilosis biofilm demonstrated increased metabolic activity ( $24 \%, P=0.024)$ compared with the controls (Table 1).

Despite the fact that direct comparisons of data from the c.f.u. assay and XTT reduction assay cannot easily be made, we compared the percentage modulation of candidal biofilm growth in the presence of E. coli and E. coli LPS in pooled data and found that there was no significant difference between the percentage modulation of Candida biofilms in the presence of E. coli or its LPS at any given time point.

\section{CLSM}

In $24 \mathrm{~h}$ dual-species biofilms, growth suppression of Candida by E. coli was clearly seen by CLSM with the Live/Dead stain, confirming the c.f.u. data. Thus, a few isolated C. krusei blastospores were seen among the cellular debris after $24 \mathrm{~h}$ (Fig. 2d) in contrast to its monospecies counterpart (Fig. 2c). Similarly, sparsely distributed $C$. dubliniensis blastospores were noted in dual-species biofilms (Fig. 2f) with E. coli after $48 \mathrm{~h}$, confirming the above quantitative findings. Some dead cells and cellular debris were also visible compared with the dense monospecies biofilm growth of the C. dubliniensis control (Fig. 2e).

With regard to E. coli, CLSM with Live/Dead stain indicated a reduction in $E$. coli cell numbers when incubated with $C$. albicans for $90 \mathrm{~min}$, confirming the c.f.u. assay data. A considerable proportion of dead E. coli cells were visible in these dual-species biofilms (Fig. 2a, b).

When the relative proportions were quantified using image analysis software, all monospecies biofilms demonstrated a higher live: dead cell ratio compared with their dualspecies counterparts (data not shown). This indicated quantitatively that there were fewer live cells and a higher number of dead cells in dual-species biofilms than in the monospecies biofilm.

\section{SEM}

In general, single-species biofilms of all Candida species grew well on the substrate on SEM observation, although species-specific growth variations were noted (Fig. 3). Thus, during the initial colonization phase of $24 \mathrm{~h}$ and the maturation phase of $48 \mathrm{~h}$, single-species yeast biofilms characteristically exhibited profuse, dense growth, although extracellular matrix was scarcely visible (Fig. 3e). In contrast, at all stages of biofilm formation, dual-species biofilms demonstrated moderate growth with a reduction in the cellularity of the biofilm. Thus, in general, the dualspecies biofilm had a reduced number of cell layers, a higher degree of cellular debris and had degrading yeast cells (Fig. 3b, d, f).

Table 1. Mean XTT values ( $\pm S D$ ) of test (E. coli LPS-treated) and control (LPS-untreated) samples of different Candida species at $90 \mathrm{~min}, 24 \mathrm{~h}$ and $48 \mathrm{~h}$

Significant differences $(P<0.05)$ are shown in bold.

\begin{tabular}{|lcccc|}
\hline Candida species & Time interval & Test $(+\mathbf{L P S})$ & Control $(-\mathbf{L P S})$ & $\boldsymbol{P}$ value \\
\hline C. albicans & $90 \mathrm{~min}$ & $0.579 \pm 0.091$ & $0.569 \pm 0.094$ & 0.795 \\
& $24 \mathrm{~h}$ & $0.736 \pm 0.142$ & $0.688 \pm 0.166$ & 0.453 \\
C. glabrata & $48 \mathrm{~h}$ & $1.251 \pm 0.041$ & $1.205 \pm 0.099$ & 0.312 \\
& $90 \mathrm{~min}$ & $0.781 \pm 0.300$ & $0.706 \pm 0.238$ & 0.862 \\
& $24 \mathrm{~h}$ & $0.920 \pm 0.316$ & $0.961 \pm 0.303$ & 0.326 \\
C. tropicalis & $48 \mathrm{~h}$ & $1.268 \pm 0.132$ & $1.237 \pm 0.082$ & 0.453 \\
& $90 \mathrm{~min}$ & $0.311 \pm 0.015$ & $0.322 \pm 0.021$ & 0.149 \\
C. parapsilosis & $24 \mathrm{~h}$ & $\mathbf{0 . 3 6 2} \pm \mathbf{0 . 0 5 5}$ & $\mathbf{0 . 3 0 3} \pm \mathbf{0 . 0 3 7}$ & $\mathbf{0 . 0 0 8}$ \\
& $48 \mathrm{~h}$ & $0.400 \pm 0.094$ & $0.410 \pm 0.051$ & 0.507 \\
C. dubliniensis & $90 \mathrm{~min}$ & $0.232 \pm 0.046$ & $0.254 \pm 0.066$ & 0.248 \\
& $24 \mathrm{~h}$ & $0.525 \pm 0.250$ & $0.412 \pm 0.107$ & 0.862 \\
C. krusei & $48 \mathrm{~h}$ & $\mathbf{1 . 0 5 3} \pm \mathbf{0 . 2 2 0}$ & $\mathbf{0 . 8 5 1} \pm \mathbf{0 . 2 6 8}$ & $\mathbf{0 . 0 2 4}$ \\
& $90 \mathrm{~min}$ & $0.318 \pm 0.032$ & $0.331 \pm 0.021$ & 0.453 \\
& $24 \mathrm{~h}$ & $\mathbf{0 . 5 6 6} \pm \mathbf{0 . 0 0 8}$ & $\mathbf{0 . 5 8 8} \pm \mathbf{0 . 0 2 3}$ & $\mathbf{0 . 0 0 4}$ \\
& $48 \mathrm{~h}$ & $0.854 \pm 0.171$ & $0.906 \pm 0.107$ & 0.402 \\
& $90 \mathrm{~min}$ & $0.656 \pm 0.169$ & $0.537 \pm 0.137$ & 0.106 \\
& $24 \mathrm{~h}$ & $1.075 \pm 0.353$ & $1.024 \pm 0.313$ & 0.862 \\
& $48 \mathrm{~h}$ & $1.367 \pm 0.101$ & $1.342 \pm 0.081$ & 0.644 \\
\hline
\end{tabular}


(a)

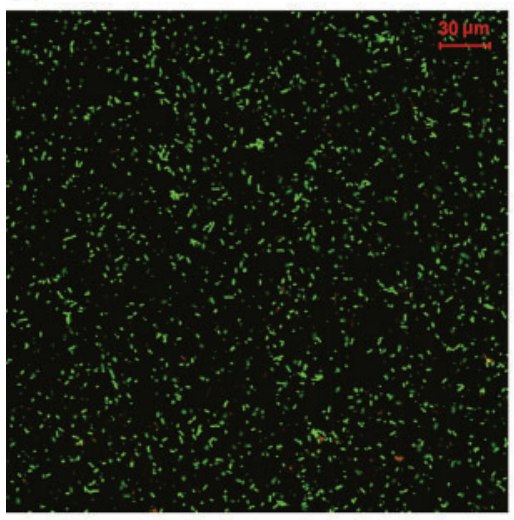

(c)

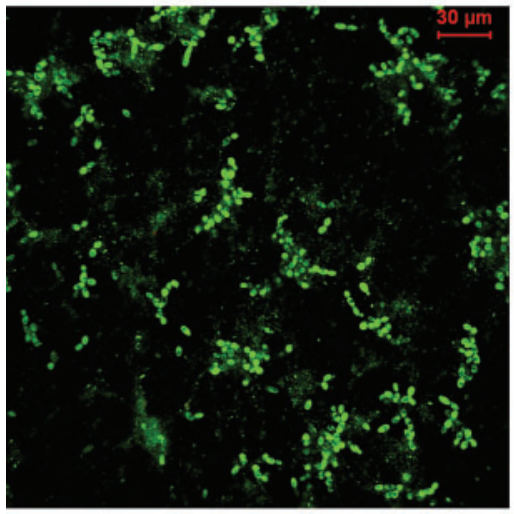

(e)

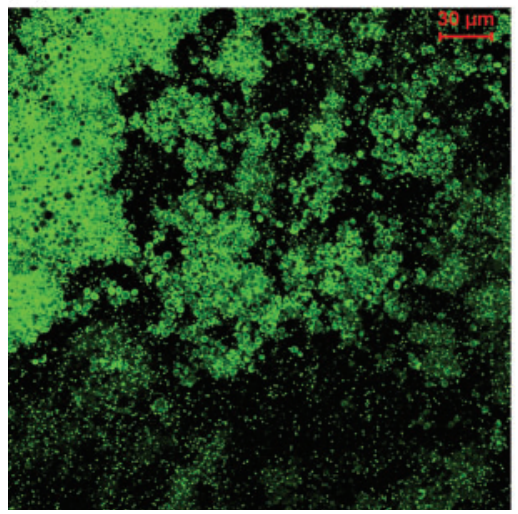

(b)

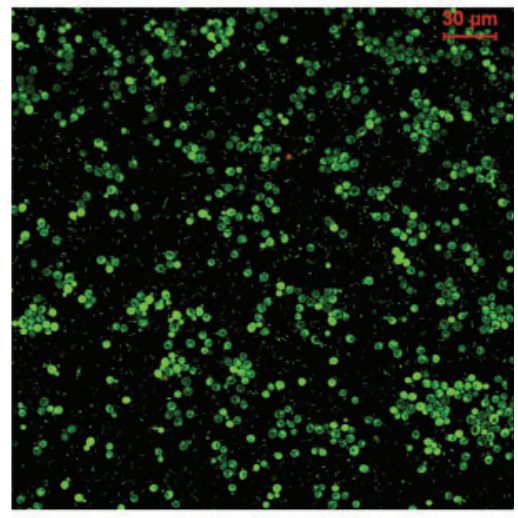

(d)

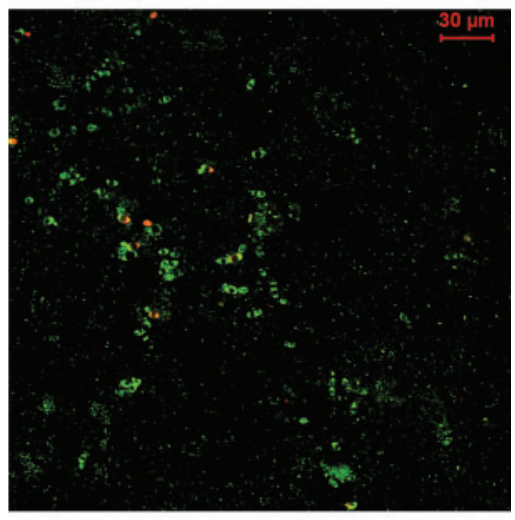

(f)

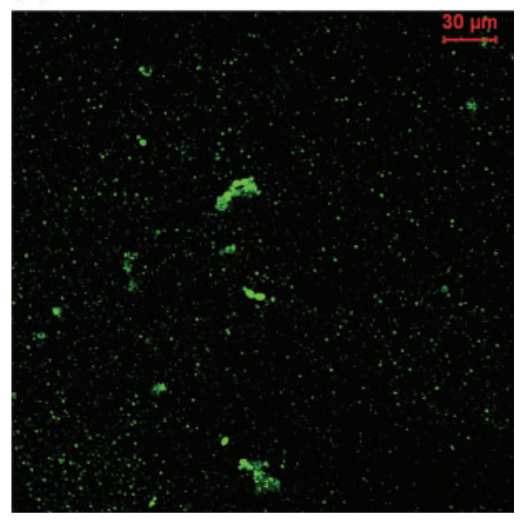

Fig. 2. CLSM images of monospecies (Candida species or E. coli) and dual species (Candida species and E. coli). Live cells are stained green and dead cells are red. (a) Adhesion of $E$. coli for 90 min. (b) Adhesion of $E$. coli in the presence of $C$. albicans for $90 \mathrm{~min}$. Note the inhibition of $E$. coli adhesion by $C$. albicans in comparison with the monospecies counterpart. (c) Initial colonization of C. krusei for 24 h. (d) Initial colonization of C. krusei co-cultured with E. coli for 24 h. Note the inhibition of C. krusei colonization by $E$. coli compared with the C. krusei monospecies biofilm. (e) Maturation of C. dubliniensis for 48 h. (f) Maturation of C. dubliniensis when co-cultured with E. coli for $48 \mathrm{~h}$. Note the suppression of $C$. dubliniensis maturation by $E$. coli in the dual-species biofilm.
With regard to E. coli, a few bacteria were seen and their density varied depending on the Candida species present and the different time intervals, and they had a less nondescript structure in comparison with the monospecies counterpart (Fig. 3d). Furthermore, the ability of $C$. albicans to suppress $E$. coli colonization in dual-species biofilms was clearly evident from SEM data (Fig. 3c, d).

\section{DISCUSSION}

Candida is a commensal yeast that colonizes oral, intestinal and vaginal mucosae of humans. The prevalence of
Candida species in oral cavities of healthy adults is approximately $50 \%$ (Samaranayake \& MacFarlane, 1990) and $C$. albicans is the most common species followed by $C$. tropicalis. However, other Candida species such as $C$. krusei, C. tropicalis, C. glabrata and C. parapsilosis are also associated with oral infections (Samaranayake et al., 1987; Webb et al., 1998). Candida can colonize and develop into biofilms on animate as well as inanimate surfaces such as acrylic dentures, stents, shunts and prostheses (e.g. voice, heart valve, knee), implants (e.g. lens, breast, denture), endotracheal tubes, pacemakers and various types of catheter (Cardinal et al., 1996; Gilbert et al., 1996; 
(a)

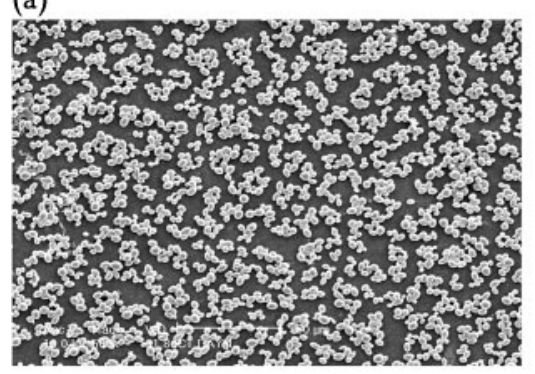

(c)

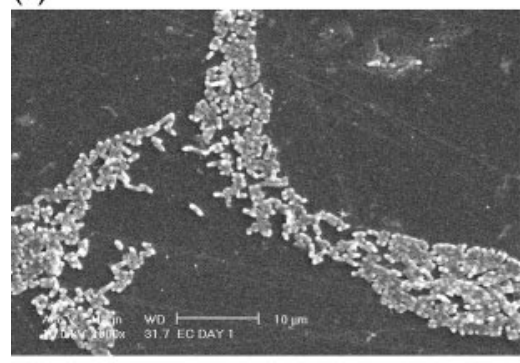

(e)

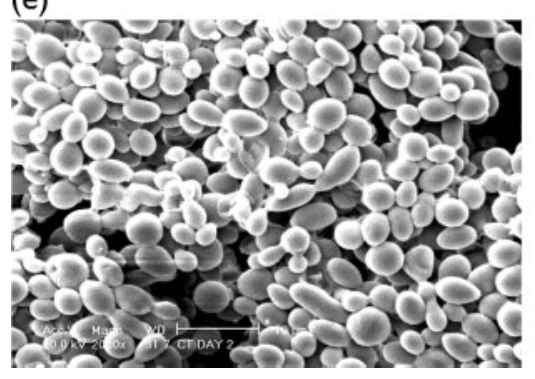

(b)

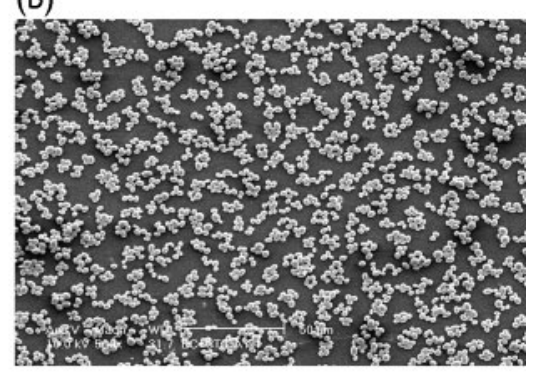

(d)

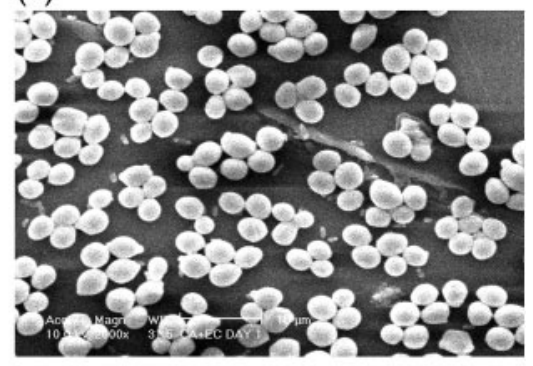

(f)

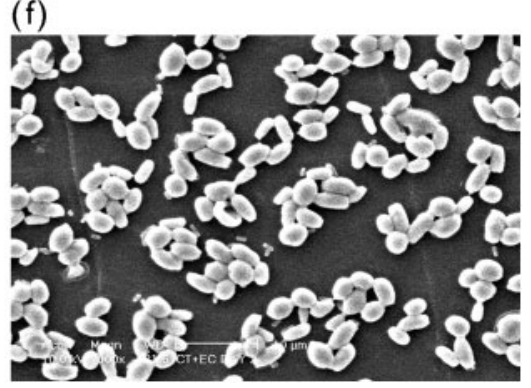

Fig. 3. SEM images of monospecies (Candida species or E. coli) and dual species (Candida species and E. coli). (a) Adhesion of C. tropicalis (90 min). (b) Adhesion of $C$. tropicalis co-cultured with E. coli (90 min). Note that $C$. tropicalis cells are smaller in size and less populous in comparison with the monospecies counterpart. (c) Adhesion of $E$. coli $(90 \mathrm{~min})$. (d) Adhesion of $E$. coli in the presence of $C$. albicans (90 min). Note that $E$. coli cell numbers are reduced and scantily distributed in comparison with the monospecies counterpart, and some cells are attached to Candida blastospores. (e) Initial colonization of C. tropicalis (24 h). (f) Initial colonization of C. tropicalis when co-cultured with $E$. coli $(24 \mathrm{~h})$. The C. tropicalis biofilm was less organized with a scanty distribution of cells in the dual-species biofilm, and some degrading yeast cells were also present. Magnification: $\times 500(a, b) ; \times 2000(c-f)$.
Goldmann \& Pier, 1993; Joly et al., 1997; Leonhardt et al., 1999; Penk \& Pittrow, 1999; Reid et al., 1992; Sen et al., 1997).

Previous studies have shown that enterobacteria including E. coli are transient colonizers of the oral cavity and are more prevalent in Asian than in Western populations (10$15 \%$ ) (Samaranayake et al., 1989; Sedgley \& Samaranayake, 1994). E. coli is particularly prevalent in the mouth as a successional community during antibiotic therapy and also in immunocompromised patients and hospitalized patients. Despite the frequent association between Candida and E. coli, few data are available on the communal behaviour of these two organisms.

The principal aim of this study, therefore, was to evaluate the qualitative and quantitative effects of the transient oral colonizer $E$. coli on various stages of in vitro biofilm development by six different species of Candida. Our results indicated that both Candida and E. coli mutually modulate biofilm development to varying degrees at different stages of biofilm formation.

Using a c.f.u. assay, we report here, for the first time, the quantitative effect of E. coli on six different Candida species.
Our results indicated that $E$. coli had no significant effect on the adhesion of $C$. albicans to polystyrene surfaces, confirming the previous results of Nair \& Samaranayake (1996a). They also demonstrated the enhanced adhesion of Candida to acrylic surfaces in the presence of E. coli ATCC 25922, irrespective of the bacterial load on the surface, and suppression of C. krusei adhesion in the presence of a high concentration of the coliform $\left(10^{5}\right.$ and $10^{6}$ cells $\left.\mathrm{ml}^{-1}\right)$. Our results extend their work, as we noted that $E$. coli suppressed the adhesion of two other relatively common Candida species, C. tropicalis and C. parapsilosis. This implies that Candida-E. coli interactions related to adhesion and aggregation may be species-specific rather than strainrelated. Further work with multiple strains of Candida from different species is required to confirm these findings.

Our results also implied that, on further prolonged incubation during the post-adhesion and biofilm development phase, E. coli suppresses the growth of C. krusei, C. parapsilosis (at $24 \mathrm{~h}$ ) and C. dubliniensis (at both 24 and 48 h). For C. albicans, although Thein et al. (2006) noted a significant inhibition of this yeast at high concentrations of E. coli $\left(2.5 \times 10^{6}, 5 \times 10^{6}\right.$ and $1 \times 10^{7}$ c.f.u. $\left.\mathrm{ml}^{-1}\right)$ at $24 \mathrm{~h}$, we were unable to confirm this finding. 
In the second part of this study, we evaluated the effect of E. coli LPS at different stages of biofilm formation on six different Candida species. Although the possible effect of LPS on the cellular layers of the biofilm is difficult to analyse with an XTT reduction assay, we tested the effect of LPS on three different growth phases of Candida biofilm development at $90 \mathrm{~min}, 24 \mathrm{~h}$ and $48 \mathrm{~h}$, as these partially reflect the metabolic activity of the growth stages in the biofilm. Palma et al. (1992) were the first to study the anticandidal effect of E. coli LPS in vitro, where graded concentrations of each LPS isolated from the cell walls of $E$. coli, Serratia marcescens and Salmonella typhimurium were added to neutrophils before addition of the target cells $(C$. albicans). They noted that LPS over a dose range of 1$1000 \mathrm{ng} \mathrm{ml}^{-1}$ strongly enhanced the anti-candidal activity of human neutrophils, although LPS itself had no direct effect on candidal growth at any concentration used. In contrast, we have reported here that the LPS of E. coli had a direct modulatory effect on in vitro biofilm formation of specific Candida species, leading to growth stimulation of C. tropicalis and C. parapsilosis and inhibition of $C$. dubliniensis. This stimulatory/suppressive effect could be due to altered cell numbers or altered cellular activity in the formed biofilm, or both. However, such growth stimulatory/suppressive effects appeared to be related to the maturation period of the biofilm. More research is required to clarify this phenomenon.

Ultrastructural views of both monospecies and dual-species biofilms confirmed the results obtained from the c.f.u. assay. Generally, all monospecies biofilms of Candida were dense, confluent, relatively well defined and uniformly distributed, with minimal extracellular polymeric materials. In contrast, yeast growth in dual-species biofilms was less dense with cell clumps, dead cells and cellular debris indicating the effect of E. coli. In addition to the reduction in overall cell counts of Candida in dual-species biofilms with E. coli, all monospecies biofilms demonstrated a higher live: dead cell ratio compared with their dualspecies counterparts on CLSM fluorescence image analysis. The dual-species biofilms were less viable than their monospecies variants, confirming the mutual inhibitory effect of Candida and E. coli during biofilm development.

A few workers have interpreted the clinical importance of such findings. Hummel et al. (1975), for instance, noted that many Gram-negative organisms including Pseudomonas and E. coli isolated from burns had a degree of inhibitory effect on C. albicans, although the effect of $E$. coli was the most consistent and reproducible. They also found that this activity was fungistatic and reversible.

In vitro studies have revealed that certain Gram-negative gut-associated organisms are frequently inhibitory towards C. albicans (Hummel et al., 1975). Approximately $20 \%$ of E. coli strains were consistently inhibitory to the growth of C. albicans. These inhibitory E. coli were constantly more sensitive to a broad range of antibiotics than their noninhibitory counterparts. Antibiotic therapy, therefore, may encourage the overgrowth of fungi not only by reducing the number of Gram-negative organisms but also by selectively eliminating those that are most suppressive to fungal growth (Hummel et al., 1975). Furthermore, viable E. coli and E. coli LPS may enhance the virulence of parenteral C. albicans, although there is a probable protective function of LPS in Candida infections initiated via the oral route (Henry-Stanley et al., 2003).

In summary, our studies focused principally on the interactions of Candida and E. coli during different stages of biofilm development and indicate that $E$. coli has a significant growth inhibitory effect on specific Candida species at various stages of biofilm development. It is also clear that some Candida species are able to withstand this effect of $E$. coli. We also revealed the mutual modulatory effects between Candida and E. coli and its LPS. Further work is necessary to explain the molecular basis of these interactions and to understand the pathobiology of mixed bacterial-fungal interactions.

\section{ACKNOWLEDGEMENTS}

The authors would like to acknowledge Dr Zaw Moe Thein for his advice. This study was supported by grant CERG HKU 7624/06M from The University of Hong Kong.

\section{REFERENCES}

Akagawa, G., Abe, S. \& Yamaguchi, H. (1995). Mortality of Candida albicans-infected mice is facilitated by superinfection of Escherichia coli or administration of its lipopolysaccharide. J Infect Dis 171, 15391544.

Banerjee, S. N., Emori, T. G., Culver, D. H., Gaynes, R. P., Jarvis, W. R., Horan, T., Edwards, J. R., Tolson, J., Henderson, T. \& Martone, W. J. (1991). Secular trends in nosocomial primary bloodstream infections in the United States, 1980-1989. National Nosocomial Infections Surveillance System. Am J Med 91, 86S-89S.

Cardinal, E., Braunstein, E. M., Capello, W. N. \& Heck, D. A. (1996). Candida albicans infection of prosthetic joints. Orthopedics 19, 247-251.

Carlsson, J. (1997). Bacterial metabolism in dental biofilms. Adv Dent Res 11, 75-80.

Cohn, Z. A. (1978). Activation of mononuclear phagocytes: fact, fancy, and future. J Immunol 121, 813-816.

Crump, J. A. \& Collignon, P. J. (2000). Intravascular catheterassociated infections. Eur J Clin Microbiol Infect Dis 19, 1-8.

Dahinden, C., Galanos, C. \& Fehr, J. (1983). Granulocyte activation by endotoxin. I. Correlation between adherence and other granulocyte functions, and role of endotoxin structure on biologic activity. $J$ Immunol 130, 857-862.

Douglas, L. J. (2003). Candida biofilms and their role in infection. Trends Microbiol 11, 30-36.

Gilbert, H. M., Peters, E. D., Lang, S. J. \& Hartman, B. J. (1996). Successful treatment of fungal prosthetic valve endocarditis: case report and review. Clin Infect Dis 22, 348-354.

Goldmann, D. A. \& Pier, G. B. (1993). Pathogenesis of infections related to intravascular catheterization. Clin Microbiol Rev 6, 176-192.

Henricks, P. A., van der Tol, M. E., Thyssen, R. M., van Asbeck, B. S. \& Verhoef, J. (1983). Escherichia coli lipopolysaccharides diminish and 
enhance cell function of human polymorphonuclear leukocytes. Infect Immun 41, 294-301.

Henry-Stanley, M. J., Hess, D. J., Erickson, E. A., Garni, R. M. \& Wells, C. L. (2003). Effect of lipopolysaccharide on virulence of intestinal Candida albicans. J Surg Res 113, 42-49.

Hummel, R. P., Maley, M. P., Miskell, P. W. \& Altemeier, W. A. (1975). Suppression of Candida albicans by Escherichia coli. J Trauma 15, 413-418.

Jin, Y., Samaranayake, L. P., Samaranayake, Y. \& Yip, H. K. (2004). Biofilm formation of Candida albicans is variably affected by saliva and dietary sugars. Arch Oral Biol 49, 789-798.

Jin, Y., Zhang, T., Samaranayake, Y. H., Fang, H. H., Yip, H. K. \& Samaranayake, L. P. (2005). The use of new probes and stains for improved assessment of cell viability and extracellular polymeric substances in Candida albicans biofilms. Mycopathologia 159, 353-360.

Joly, V., Belmatoug, N., Leperre, A., Robert, J., Jault, F., Carbon, C. \& Yeni, P. (1997). Pacemaker endocarditis due to Candida albicans: case report and review. Clin Infect Dis 25, 1359-1362.

Jones, R. N., Marshall, S. A., Pfaller, M. A., Wilke, W. W., Hollis, R. J., Erwin, M. E., Edmond, M. B. \& Wenzel, R. P. (1997). Nosocomial enterococcal blood stream infections in the SCOPE Program: antimicrobial resistance, species occurrence, molecular testing results, and laboratory testing accuracy. SCOPE Hospital Study Group. Diagn Microbiol Infect Dis 29, 95-102.

Leonhardt, A., Renvert, S. \& Dahlen, G. (1999). Microbial findings at failing implants. Clin Oral Implants Res 10, 339-345.

Lewis, K. (2001). Riddle of biofilm resistance. Antimicrob Agents Chemother 45, 999-1007.

Morrison, D. C. \& Ryan, J. L. (1987). Endotoxins and disease mechanisms. Annu Rev Med 38, 417-432.

Nair, R. G. \& Samaranayake, L. P. (1996a). The effect of oral commensal bacteria on candidal adhesion to denture acrylic surfaces. An in vitro study. APMIS 104, 339-349.

Nair, R. G. \& Samaranayake, L. P. (1996b). The effect of oral commensal bacteria on candidal adhesion to human buccal epithelial cells in vitro. J Med Microbiol 45, 179-185.

Nobile, C. J. \& Mitchell, A. P. (2007). Microbial biofilms: e pluribus unum. Curr Biol 17, R349-R353.

Odds, F. C. (1987). Candida infections: an overview. Crit Rev Microbiol 15, 1-5.

Palma, C., Cassone, A., Serbousek, D., Pearson, C. A. \& Djeu, J. Y. (1992). Lactoferrin release and interleukin-1, interleukin-6, and tumor necrosis factor production by human polymorphonuclear cells stimulated by various lipopolysaccharides: relationship to growth inhibition of Candida albicans. Infect Immun 60, 4604-4611.
Penk, A. \& Pittrow, L. (1999). Role of fluconazole in the long-term suppressive therapy of fungal infections in patients with artificial implants. Mycoses 42 (Suppl. 2), 91-96.

Pfaller, M. A., Jones, R. N., Messer, S. A., Edmond, M. B. \& Wenzel, R. P. (1998). National surveillance of nosocomial blood stream infection due to Candida albicans: frequency of occurrence and antifungal susceptibility in the SCOPE Program. Diagn Microbiol Infect Dis 31, 327-332.

Potera, C. (1999). Forging a link between biofilms and disease. Science 283, 1837-1839.

Ramage, G., Vandewalle, K., Wickes, B. L. \& López-Ribot, J. L. (2001). Characteristics of biofilm formation by Candida albicans. Rev Iberoam Micol 18, 163-170.

Reid, G., Denstedt, J. D., Kang, Y. S., Lam, D. \& Nause, C. (1992). Microbial adhesion and biofilm formation on ureteral stents in vitro and in vivo. J Urol 148, 1592-1594.

Rietschel, E. T., Schade, U., Jensen, M., Wollenweber, H. W., Luderitz, O. \& Greisman, S. G. (1982). Bacterial endotoxins: chemical structure, biological activity and role in septicaemia. Scand J Infect Dis Suppl 31, 8-21.

Samaranayake, L. P. (2006). Essential Microbiology for Dentistry, 3rd edn. Edinburgh: Churchill Livingstone.

Samaranayake, L. P. \& MacFarlane, T. W. (1990). Oral Candidosis. London: Wright.

Samaranayake, L. P., MacFarlane, T. W. \& Williamson, M. I. (1987). Comparison of Sabouraud dextrose and Pagano-Levin agar media for detection and isolation of yeasts from oral samples. J Clin Microbiol $25,162-164$.

Samaranayake, L. P., Lamb, A. B., Lamey, P. J. \& MacFarlane, T. W. (1989). Oral carriage of Candida species and coliforms in patients with burning mouth syndrome. J Oral Pathol Med 18, 233-235.

Sedgley, C. M. \& Samaranayake, L. P. (1994). The oral prevalence of aerobic and facultatively anaerobic Gram-negative rods and yeasts in Hong Kong Chinese. Arch Oral Biol 39, 459-466.

Sen, B. H., Safavi, K. E. \& Spangberg, L. S. (1997). Colonization of Candida albicans on cleaned human dental hard tissues. Arch Oral Biol 42, 513-520.

Thein, Z. M., Samaranayake, Y. H. \& Samaranayake, L. P. (2006). Effect of oral bacteria on growth and survival of Candida albicans biofilms. Arch Oral Biol 51, 672-680.

Webb, B. C., Thomas, C. J., Willcox, M. D., Harty, D. W. \& Knox, K. W. (1998). Candida-associated denture stomatitis. Aetiology and management: a review. Part 2. Oral diseases caused by Candida species. Aust Dent J 43, 160-166. 